\title{
Across-Site Threshold Variation in Cochlear Implants: Relation to Speech Recognition
}

\author{
Bryan E. Pfingst $^{\mathrm{a}} \mathrm{Li} \mathrm{Xu}^{\mathrm{a}, \mathrm{b}}$ Catherine S. Thompson ${ }^{\mathrm{a}}$ \\ ${ }^{a}$ Department of Otolaryngology, Kresge Hearing Research Institute, University of Michigan Health System, \\ Ann Arbor, Mich., and ${ }^{\mathrm{b}}$ School of Hearing, Speech and Language Sciences, Ohio University, Athens, Ohio, USA
}

\section{Key Words}

Cochlear implant · Auditory prosthesis · Detection

thresholds · Speech recognition · Human

\begin{abstract}
Functional implications of across-site variation in detection thresholds in subjects with cochlear implants were evaluated by comparing thresholds to speech recognition performance. Detection thresholds for bipolar (BP) and monopolar (MP) stimulation of all available stimulation sites were assessed in 21 subjects with $\mathrm{Nu}$ cleus ${ }^{\circledR} \mathrm{Cl} 24 \mathrm{M}$ and $\mathrm{Cl} 24 \mathrm{R}(\mathrm{CS})$ implants. We found significant negative correlations between speech recognition and within-subject across-site threshold variance for both BP and MP stimulation, but no significant correlation of speech recognition with mean threshold levels. These results suggest that across-site variance of detection thresholds could provide a useful early indication of the prognosis for speech recognition and might serve as an indicator for specific therapeutic approaches in individual subjects.
\end{abstract}

Copyright $\subset 2004$ S. Karger AG, Basel

\section{KARGER}

(c) 2004 S. Karger AG, Basel

Fax +4161306 1234 E-Mail karger@karger.ch www.karger.com www.karger.com/aud

\section{Introduction}

A high degree of variability in speech recognition performance is found in patients with cochlear implants [Pfingst, 2000; Skinner et al., 2002]. There is little doubt that multiple factors, ranging from biophysical to neural, to cognitive, contribute to this variability [Blamey et al., 1996; Kawano et al., 1998; Kileny et al., 1991; Knutson et al., 1991; Rubinstein et al., 1999]. Diagnostic tools are needed to assess the origins of this variability and thus guide the clinician in selecting therapeutic strategies for individual patients. In this paper, we consider psychophysical detection thresholds as potential diagnostic indicators.

Detection thresholds have several potential advantages in this context. First, the measures are noninvasive and easy to obtain. Second, they are used commonly with cochlear implant patients for fitting purposes, so subjects have experience with the testing procedures. The results are typically reliable. Finally, since the measurement procedure requires little cognitive skill on the part of the listener, thresholds are likely to reflect physical and physiological variability among patients and among stimulation sites within a given individual.

A primary focus of this paper is on variation in psychophysical detection thresholds across stimulation sites

Bryan E. Pfingst, $\mathrm{PhD}$

University of Michigan, Kresge Hearing Research Institute

1301 E. Ann Street

Ann Arbor, MI 48109 (USA)

Tel. +1 734763 2292, Fax +1 734764 0014, E-Mail bpfingst@umich.edu 
within individual subjects. In a previous study [Pfingst and $\mathrm{Xu}, 2004]$, we examined the variation in psychophysical detection thresholds ( $\mathrm{T}$ levels) and maximum comfortable loudness levels (C levels) that occur from one stimulation site to the next across the 22-electrode implant in subjects with Nucleus ${ }^{\circledR}$ cochlear implants. We found that (1) across-site variation in these measures was much larger for narrow bipolar (BP) stimulation than for monopolar (MP) stimulation and (2) the magnitude and pattern of variation across sites for $\mathrm{C}$ levels were roughly comparable to those for T levels. We concluded that these results were compatible with the hypothesis that acrosssite variation results in large part from variation in the distance between the electrodes and the sites of action potential initiation. These distances would be expected to vary as a function of the position of the electrodes in the scala tympani, the presence of fibrous tissue and new bone in the scala tympani, and as a function of neuropathology. Distance is an important determinant of threshold level because activating potentials decrease as a function of distance from the electrodes. Neuropathology can create large variation in the distances between the electrodes and the sites of action potential initiation because it can affect both the location and the excitability of neurons along the length of the cochlear spiral. For example, if neurons near the stimulating electrodes are dead or if they have high thresholds for electrical stimulation, this might result in the most sensitive site for activation being remote from the electrodes. Neuropathology could also create variation from site to site in the sensitivity of the neurons that are activated by a given stimulus site, giving rise to additional across-site variability.

In the present study, we examined the functional significance of across-site (within-subject) variation and across-subject variation in detection thresholds by examining the relationship between thresholds and speech recognition for multichannel processing strategies that used most or all of the 22-electrode array. Based on the considerations discussed above, we derived the following hypotheses.

One hypothesis was based on the assumption that siteto-site variation in psychophysical detection thresholds is due, in part, to variation in neuropathology along the length of the cochlear spiral. We further assumed that variability in nerve survival would be detrimental to speech recognition with a cochlear prosthesis for a variety of reasons, including distortion of the place pitch code and variation in the number of neurons carrying information from each channel of the prosthesis. Based on these assumptions, we hypothesized that the magnitude of within-subject across-site variation in detection thresholds would be correlated with speech recognition performance with cochlear implants.

A second hypothesis was based on similar assumptions. We assumed that the relative levels of detection thresholds at various sites along the electrode array reflect the distance between the electrodes and the sites of action potential initiation and the condition of the stimulated neurons; i.e., that lower psychophysical detection thresholds reflect closer proximity between electrodes and sites of action potential initiation and healthier neurons near the electrodes. We assumed that achieving a close proximity between electrodes and sites of action potential initiation would result in better channel separation and consequently better speech recognition with a multichannel prosthesis and that neurons with low activation thresholds would be healthier and more able to accurately encode information represented in the electrical stimuli. These assumptions led to the hypothesis that lower average detection thresholds across all stimulation sites in a subject's implant would be correlated across subjects with better speech recognition.

Finally, we considered the effects of stimulus waveform, specifically pulse phase duration, on the across-site variation measures. Neural models and physiological experiments have demonstrated that significant changes in temporal integration properties of the neural population activated by electrical stimulation can occur when the site of action potential initiation is altered by pathology [Colombo and Parkins, 1987; van den Honert and Stypulkowski, 1984]. These changes can be reflected in the slopes of strength-duration functions (threshold for activation as a function of stimulus phase duration) because of variation in membrane properties (current integration) among the various activation sites. If the slopes of the strength-duration functions for two sites of activation are different, then the differences in threshold between those sites will vary as a function of stimulus phase duration. These considerations led to the hypothesis that the magnitude of across-site variation in a cochlear implant would depend on the pulse duration.

\section{Methods}

Subjects

Twenty-one postlingually deaf adult human subjects participated in the study. Fifteen had Nucleus ${ }^{\circledR}$ CI24R(CS) (Contour ${ }^{\mathrm{TM}}$ ) implants and 6 had Nucleus CI24M (straight array) implants. All subjects spoke American English as their native language. All had at least 8 months of experience with the implants prior to the onset 
Table 1. Subject characteristics

\begin{tabular}{|c|c|c|c|c|c|c|c|}
\hline $\begin{array}{l}\text { Subject } \\
\text { code }\end{array}$ & $\begin{array}{l}\text { Age at } \\
\text { testing } \\
\text { years }\end{array}$ & $\begin{array}{l}\text { Age at } \\
\text { onset of } \\
\text { profound } \\
\text { deafness } \\
\text { years }\end{array}$ & $\begin{array}{l}\text { Duration } \\
\text { of deafness } \\
\text { prior to } \\
\text { implant } \\
\text { years }\end{array}$ & Implant type & $\begin{array}{l}\text { Normal } \\
\text { processing } \\
\text { strategy }\end{array}$ & Etiology of deafness & $\begin{array}{l}\text { PTA } \\
\text { pre-op. } \\
\text { unaided in } \\
\text { implanted } \\
\text { ear }\end{array}$ \\
\hline S1 & 56 & 47 & 4 & CI24M & SPEAK & unknown & 102 \\
\hline $\mathrm{S} 2$ & 76 & 70 & 2 & CI24M & SPEAK & scarlet fever & 103 \\
\hline $\mathrm{S} 3$ & 52 & 34 & 14 & CI24M & $\mathrm{ACE}$ & unknown & NA \\
\hline $\mathrm{S} 4$ & 80 & 47 & 30 & CI24M & $\mathrm{ACE}$ & unknown & 118 \\
\hline S5 & 62 & 37 & 23 & $\mathrm{CI} 24 \mathrm{R}(\mathrm{CS})$ & SPEAK & unknown & NR \\
\hline S6 & 68 & 36 & 30 & CI24R(CS) & SPEAK & hereditary & 100 \\
\hline S7 & 56 & 54 & 0 & CI24R(CS) & $\mathrm{ACE}$ & measles or mumps & 120 \\
\hline $\mathrm{S} 8$ & 31 & 28 & 1 & CI24R(CS) & $\mathrm{ACE}$ & unknown & 82 \\
\hline S9 & 38 & 37 & 0 & CI24R(CS) & $\mathrm{ACE}$ & unknown & 92 \\
\hline $\mathrm{S} 10$ & 46 & 37 & 8 & CI24R(CS) & $\mathrm{ACE}$ & hereditary & 117 \\
\hline S11 & 43 & 40 & 2 & CI24R(CS) & $\mathrm{ACE}$ & hereditary & 107 \\
\hline S12 & 47 & 44 & 1 & CI24R(CS) & $\mathrm{ACE}$ & trauma + existing deficit & 83 \\
\hline $\mathrm{S} 13$ & 33 & 3 & 28 & CI24R(CS) & $\mathrm{ACE}$ & hereditary & 105 \\
\hline S14 & 44 & 3 & 40 & CI24R(CS) & $\mathrm{ACE}$ & congenital & 102 \\
\hline $\mathrm{S} 15$ & 51 & 25 & 21 & $\mathrm{CI} 24 \mathrm{M}$ & $\mathrm{ACE}$ & viral infection & NR \\
\hline S16 & 55 & 17 & 30 & CI24R(CS) & $\mathrm{ACE}$ & unknown & 100 \\
\hline S17 & 68 & 30 & 37 & CI24R(CS) & ACE & $\begin{array}{l}\text { ototoxicity + existing } \\
\text { deficit }\end{array}$ & NA \\
\hline $\mathrm{S} 18$ & 43 & 27 & 11 & CI24M & SPEAK & hereditary & 100 \\
\hline S19 & 74 & 22 & 51 & CI24R(CS) & $\mathrm{ACE}$ & hereditary & 102 \\
\hline S20 & 65 & 60 & 3 & CI24R(CS) & SPEAK & $\begin{array}{l}\text { high fever + Ménière's } \\
\text { disease }\end{array}$ & NA \\
\hline $\mathrm{S} 21$ & 54 & 23 & 25 & CI24R(CS) & SPEAK & unknown & NA \\
\hline
\end{tabular}

PTA = Pure-tone average thresholds; NA = not available; NR = no response at the limits of the audiometer.

of this study. All subjects used MP electrode configurations in the processors that they wore every day. Further details for each subject are given in table 1. Preimplant characteristics (e.g. age, duration of deafness, or preoperative acoustic thresholds) did not differ appreciably between the groups of subjects with the two implant types: CI24M and CI24R(CS). In a previous study [Pfingst and Xu, 2004], we found considerable overlap in $\mathrm{T}$ levels and across-site variation in T levels between CI24M and CI24R(CS) implants. On average, $\mathrm{T}$ levels for CI24R(CS) implants were slightly lower and the acrosssite variation was slightly higher but not significantly different from the values obtained for CI24M implants.

The use of human subjects in this research was reviewed and approved by the University of Michigan Medical School Institutional Review Board.

\section{Research Design}

The dependent variables in this study were across-site variation in $\mathrm{T}$ levels, across-subject variation in mean $\mathrm{T}$ levels across the electrode array, slopes of psychophysical strength-duration functions, and speech recognition. To assess across-site variation, $\mathrm{T}$ levels were collected from all available stimulation sites in the 22electrode arrays in the 21 subjects. 'Stimulation site' was defined as the physical location of the intracochlear electrodes through which current was passed to stimulate neurons. For MP stimulation, there were 22 possible sites and for BP stimulation, there were 21. A few sites in a few subjects were not tested because the sites were not functional or because stimulation at those sites produced uncomfortable sensations.

Three metrics were used to assess across-site variation within subjects: the variance of $\mathrm{T}$ levels for all tested sites, the range of $\mathrm{T}$ levels (highest minus lowest) across all tested sites, and site-to-site variation. We defined 'site-to-site variation' as the mean of the absolute values of the differences between $\mathrm{T}$ levels measured at each stimulation site within a given electrode array and the $\mathrm{T}$ level at the more apical adjacent site. This was calculated by the formula:

$$
\text { site-to-site variation }=\frac{\sum_{i=1}^{N-1}\left|T_{i}-T_{i+1}\right|}{N-1},
$$

where $T$ is the threshold measured at a particular site $(i)$ and $N$ is the total number of sites measured. Comparisons were included only if $\mathrm{T}$ levels were obtained at two adjacent sites. Usually, data were obtained for 20 adjacent sites in the BP condition and 21 adjacent sites in the MP condition. 
For across-subject variation in T levels, we calculated the mean $\mathrm{T}$ levels for all tested sites within each subject.

Psychophysical strength-duration functions ( $T$ level versus pulse duration functions) were obtained for 7-9 sites in the electrode array. The sites selected for determination of strength-duration functions included sites with highest, lowest and mid-level thresholds for BP and for MP stimulation and sites with the largest, the smallest and a mid-sized difference between BP and MP thresholds.

Speech recognition was tested using three tests: consonant recognition, vowel recognition, and sentence recognition in background noise. Details of the speech tests are given below.

\section{Hardware and Software for Electrical Stimulation}

Two Nucleus cochlear implant designs were used: CI24R(CS) (15 subjects) and CI24M (6 subjects). Each design consisted of an array of 22 electrodes with a Silastic rubber carrier inserted into the scala tympani, and two extracochlear electrodes. The two implant designs differed in the geometry (shape, location and spacing) of the electrodes and in the shape of the Silastic rubber carrier. The CI24R(CS) implant used half-band electrodes located on only one side of the Silastic carrier and the implant was precurved to match the medial aspect of the scala tympani with the intention that, when inserted in the cochlea, it would position the electrodes near the modiolar wall. The CI24M implant used band electrodes that surrounded the Silastic carrier and the array was straight prior to insertion in the scala tympani. For both implant designs, electrodes in the scalar array were numbered from 1 to 22 with 22 being the most apical.

T levels were measured for each of two electrode configurations: BP and MP. In the BP configuration, stimulation was between pairs of adjacent electrodes in the scala tympani array. The more basal electrode in the pair was arbitrarily labeled the 'active' electrode and the more apical member of the pair was labeled the 'return' electrode. The spacing of the electrodes varied depending on the implant design. For CI24M implants, the spacing of all adjacent electrodes was approximately $0.75 \mathrm{~mm}$ center to center. For the CI24R(CS) implants, the center-to-center spacing ranged from about $0.8 \mathrm{~mm}$ at the basal end of the implant to about $0.6 \mathrm{~mm}$ at the apical end, averaging about $0.64 \mathrm{~mm}$. In the MP configuration, stimulation was between an electrode in the scala tympani array and two extracochlear electrodes in parallel. The intracochlear electrode was labeled the 'active' electrode. The extracochlear electrodes comprised a ball electrode buried underneath the temporalis muscle and a plate electrode located on the casing of the implanted receiver/stimulator.

For measurements of $\mathrm{T}$ levels, a single, laboratory-owned, SPrint ${ }^{\circledR}$ processor (serial number 408594, Cochlear Corporation, Englewood, Colo., USA) was used. The stimuli were controlled by custom software run on an IBM-compatible personal computer. This software generated sequences of frames and sent instructions to the SPrint processor utilizing Nucleus Implant Communicator ${ }^{\circledR}$ (version 3.27) software libraries. The software communicated with the SPrint processor using an IF5 ISA card and a Processor Control Interface (Cochlear Corporation). The SPrint processor controlled transmission of radio frequency pulses to subject's implanted receiver/stimulator. The implanted receiver/stimulator then decoded the radio frequency information to the correct stimulation pulse parameters and delivered appropriate current pulses to the specified sites in the implanted cochlear electrode array. The custom software also provided a user interface that was used to collect the psychophysical responses from the subjects, as detailed below.

The implanted receiver-stimulators delivered current in 256 steps, specified in current level units (CL). Currents ranged from approximately $10 \mu \mathrm{A}$ peak at $\mathrm{CL}=0$ to approximately $1750 \mu \mathrm{A}$ peak at $C L=255$ with a step size of approximately $2.046 \%(0.176$ $\mathrm{dB})$. Output of the implanted receiver-stimulators at a given CL varied from stimulator to stimulator over a range of about $\pm 10 \%$. A calibration for each of the subject's implanted receiver-stimulators was obtained from Cochlear Corporation. These calibrations were used to calculate stimulation levels in microamperes, as described previously [Pfingst and $\mathrm{Xu}, 2004$ ].

For speech recognition testing, subjects wore their own processors, which were programmed with their normal everyday processing strategies and electrode configurations (table 1). We used the processing strategies with which the subjects were most familiar in order to eliminate confounding effects of experience. The subjects sat in a double-walled sound-attenuating chamber (Acoustic Systems Model RE 242S). Speech recognition test materials were presented from sound files on a desktop computer. The signals were passed through a Rane ME-60 graphic equalizer and a Crown D-75 amplifier and presented through a TDC 4A loudspeaker positioned about $1 \mathrm{~m}$ away from the subject at about $0^{\circ}$ azimuth. The equalizer was adjusted such that the output of the speaker produced a flat spectrum when presented with white noise to assure compliance beyond ANSI 3.6 specifications.

The speech test level was calibrated periodically with a sound level meter (Brüel and Kjær Type 2231). Subjects were not present during the calibration. The sound level meter was positioned near where the headset microphone would be located during the test sessions. A fast time setting (i.e., time constant of $125 \mathrm{~ms}$ ) and an 'A' frequency weighting were set on the sound level meter during the calibration of the speech materials.

\section{Stimuli}

For T level measurements, stimuli consisted of symmetric-biphasic pulses presented at a rate of 250 pulses/s. To assess acrosssite variation, $\mathrm{T}$ levels at all testable sites were obtained using $200 \mu \mathrm{s} /$ phase pulses. For psychophysical strength-duration functions, phase durations ranged from 25 to $1600 \mu \mathrm{s} /$ phase in steps of doubling. However, for some stimulation sites in some subjects, we were unable to test the full range of phase durations due to limitations of the implanted stimulator. For all testing, the polarity of the initial phase of each pulse to the 'active' electrodes was negative and the interphase gap was $45 \mu \mathrm{s}$. The stimulus burst duration was $500 \mathrm{~ms}$. T levels were measured using both BP and MP electrode configurations.

For speech recognition testing, three sets of stimuli were used: (1) consonants, (2) vowels, and (3) Hearing in Noise Test (HINT) sentences in speech-shaped noise.

For consonant recognition tests, recordings made by Shannon et al. [1999] of 20 naturally spoken American English consonants in a consonant-/a/ context were used: ba, cha, da, fa, ga, ja, ka, la, ma, na, pa, ra, sa, sha, ta, tha, va, wa, ya, and za. Two talkers were used (male No. 3 and female No. 3 ) for a total of 40 stimuli.

For vowel recognition tests, recordings made by Hillenbrand et al. [1995] of 12 naturally spoken American English vowels in an /h/-vowel-/d/ context were used: had, hayed, hawed, head, heard, heed, hid, hod, hoed, hood, hud, and who'd. Two male (No. 48 and No. 49) and 2 female (No. 39 and No. 44) talkers were selected from 
the recordings by Hillenbrand et al., giving a total of four sets of natural /h/-vowel-/d/ tokens for a total of 48 stimuli.

For sentence-in-noise testing, the HINT created by Nilsson et al. [1994] was used. Lists of 10 sentences each were drawn at random from the 25 lists in the test set. The sentences in this test are spoken in American English and have been carefully equated for naturalness, length and intelligibility. Sentences were presented in speech-shaped noise that matched the spectra of the sentences. The signal-to-noise ratio was $10 \mathrm{~dB}$.

\section{Psychophysical Procedures}

The method of adjustment was used to measure T levels. In a previous study [Pfingst and $\mathrm{Xu}, 2004$ ], we found that thresholds measured with this method were usually slightly higher (approximately $1.7 \mathrm{~dB}$ on average) than those measured using a two-alternative forced-choice method with adaptive tracking. However, across-site variation in thresholds measured with these two methods was not significantly different.

The procedure for the method of adjustment was similar to that used in fitting implants clinically. In this procedure, the stimuli were presented repeatedly with a 500-ms on/off duty cycle. Arrow keys on the computer keyboard were used to adjust the level of the current. The subject was instructed to adjust the level of the signal until it was 'barely audible'. For this study, T levels were determined in random order from all stimulation sites in the test set. After the first set of thresholds was obtained, new randomizations were used for a second and third set of threshold measurements. Results for the three measurements at each site were then averaged.

\section{Speech Recognition Testing}

For speech recognition tests, subjects were first given a preview of the sounds and then a practice session. Then each test was administered three times. The results of the three tests were averaged. For the consonant and vowel recognition tests, the same stimuli were used for each repetition but with a new randomization each time. For the HINT sentence tests, a different list of 10 sentences was used for each of the three administrations of the test.

For the consonant and vowel recognition tests, the stimuli were presented respectively in 20- or 12-alternative forced-choice paradigms. Tokens were presented in random order without replacement. The listeners viewed a screen with the choices listed alphabetically and used a computer mouse to select their responses.

For the HINT sentence tests, subjects reported their responses verbally and the tester scored them using the score sheet provided with the test materials. The sentences were scored based on the percentage of correct words.

\section{Results}

Threshold-versus-stimulation-site functions for 3 subjects are shown in figure 1 to illustrate cases with high, medium, and low across-site threshold variation for BP and MP stimulation. This figure also shows examples of mean thresholds across the whole array, represented by triangles next to the left ordinate.

Implant Thresholds and Speech

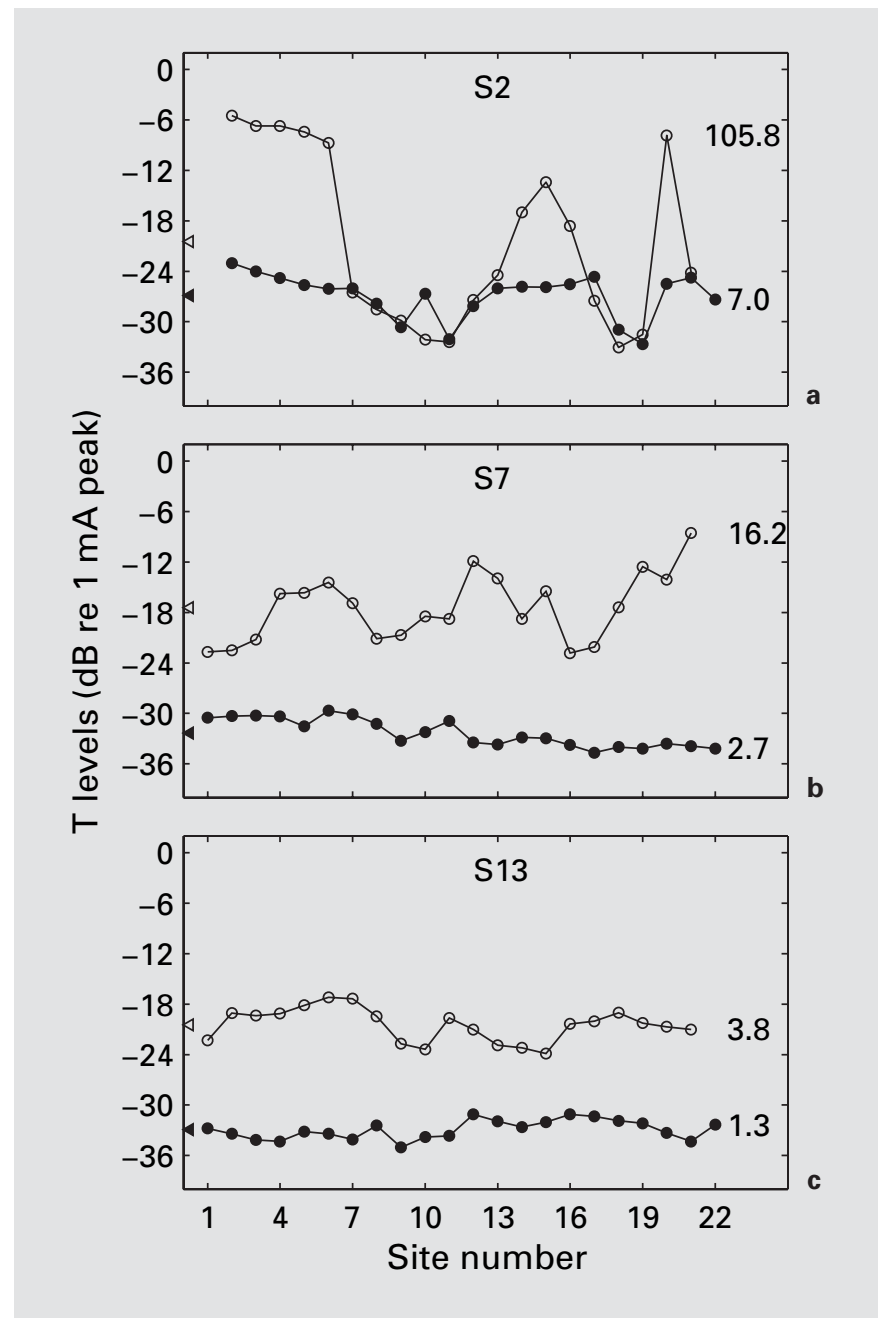

Fig. 1. Detection thresholds versus stimulation site for 3 subjects illustrating cases with high (a), medium (b) and low (c) across-site variation. Open circles are for BP stimulation and filled circles are for MP stimulation. Site number indicates the number of the active electrode with the basal end of the implant being to the left (site 1). Across-site variance $\left(\mathrm{dB}^{2}\right)$ for each function is listed to the right of each function. The mean threshold for all tested sites in each function is shown by triangles on the left ordinate using open symbols for BP and filled symbols for MP thresholds. Subject numbers are given at the top center of each panel. Consonant recognition scores for these 3 subjects were: $\mathrm{S} 2=15 \%, \mathrm{~S} 7=57 \%$ and $\mathrm{S} 13=69 \%$.

A key hypothesis examined in these studies was that high across-site variation in detection thresholds reflects conditions that are detrimental to good speech recognition with cochlear implants. Consistent with this hypothesis, we found significant negative correlations (z test; $\mathrm{p}<0.05)$ between across-site variation in $\mathrm{T}$ levels and speech recognition (fig. 2). Subjects with relatively large 
Fig. 2. Relationship between across-site variances of $\mathrm{T}$ levels (abscissa) and speech recognition scores (ordinate). Each data point represents 1 subject with open symbols for CI24M subjects and filled symbols for CI24R(CS) subjects. The left column is for BP stimulation and the right column is for MP stimulation. The three rows, top to bottom, represent the data from consonant, vowel, and HINT sentence $(10 \mathrm{~dB} \mathrm{~S} / \mathrm{N})$ tests, respectively. Linear regression lines are shown with the correlation coefficients indicated at the upper right corner of each panel. All correlations were statistically significant ( $\mathrm{z}$ test; $\mathrm{p}<0.05)$.
BP

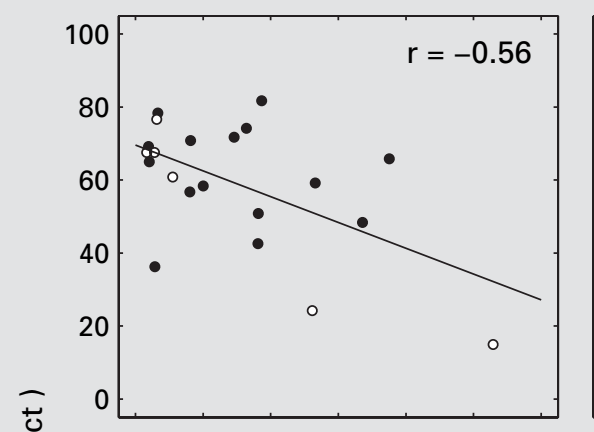

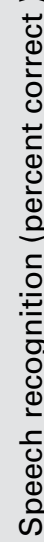

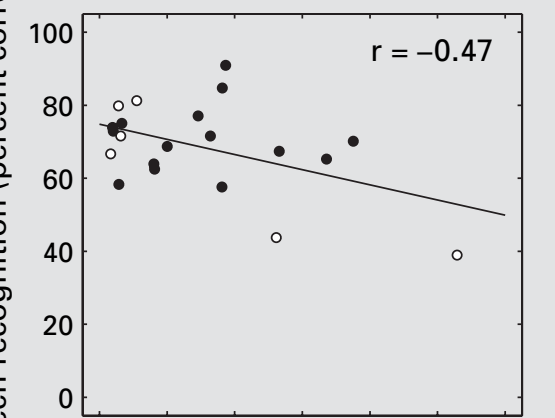

की 100

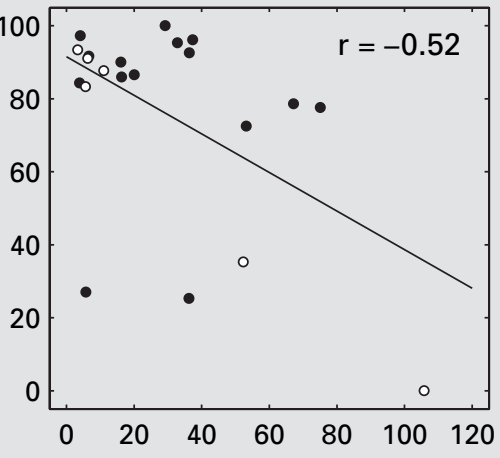

Variance across sites $\left(\mathrm{dB}^{2}\right)$
MP
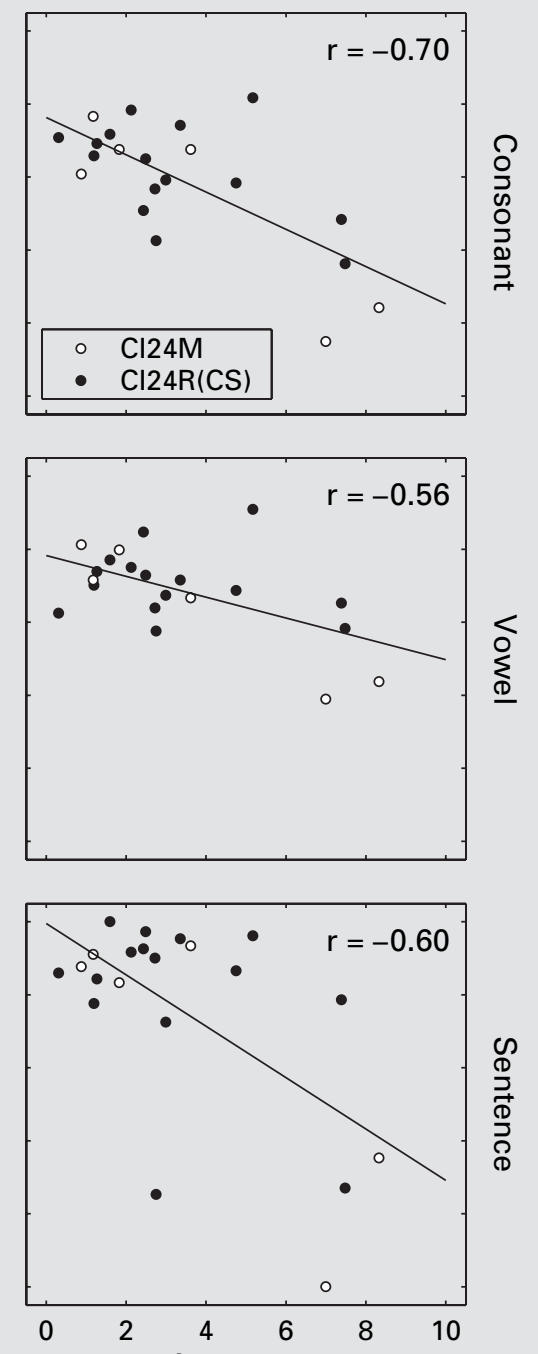

across-site variation in T levels with Nucleus $24 \mathrm{R}(\mathrm{CS})$ or $24 \mathrm{M}$ implants usually had poorer speech recognition scores. This relationship held for both BP and MP stimulation. It was slightly stronger for MP stimulation, even though across-site variation for MP stimulation was much smaller than that for BP stimulation. The relationship was evident for consonants, vowels, and HINT sentences in noise. The correlations were negative for all three measures of across-site variation and were statistically significant ( $\mathrm{z}$ test; $\mathrm{p}<0.05$ ) in all but 4 of the 18 cases (ta- ble 2). Correlation coefficients for consonants were slightly stronger than those for vowels or sentences in noise.

Since across-site variation in T levels for both BP and MP stimulation were correlated with speech recognition, we examined the correlations across the 21 subjects between across-site variances for BP stimulation and those for MP stimulation to evaluate the degree to which T levels for BP and MP stimulation reflected similar underlying mechanisms (fig. 3). Although there was a statistically significant correlation between across-site variances for BP 


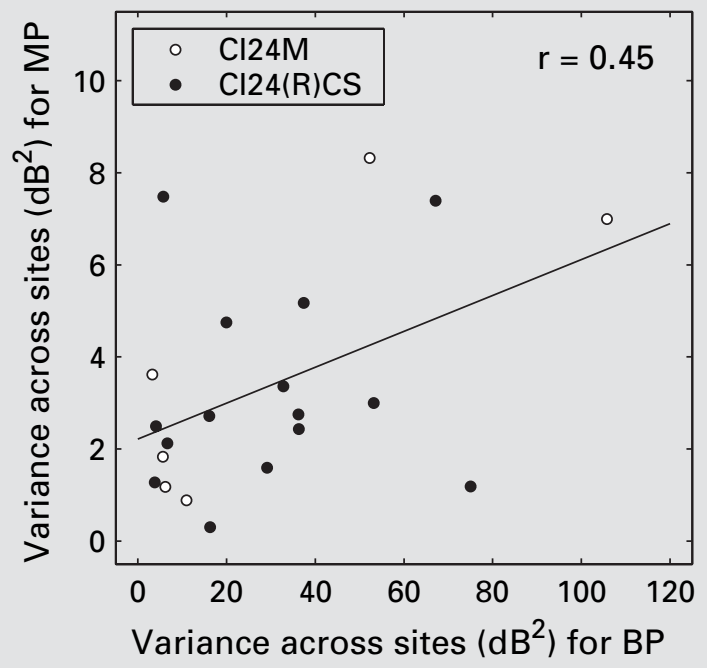

Fig. 3. Relationship between across-site variance for BP stimulation and across-site variance for MP stimulation in the same subjects. Each data point represents an individual subject with CI24M implants represented by open circles and CI24R(CS) implants represented by filled circles. A least-squares regression line for all 21 data points is shown. The correlation coefficient, shown in the upper right corner, is statistically significant ( $\mathrm{z}$ test; $\mathrm{p}<0.05$ ). stimulation and those for MP stimulation, there were cases where across-site variance for MP was high and that for $\mathrm{BP}$ was low, and vice versa. This suggests that the mechanisms underlying across-site variation in T levels for these two electrode configurations are not identical in detail. This is also evident from the examples shown in figure 1 . For $\mathrm{S} 2$, there is a weak correspondence between the acrosssite patterns for BP and MP stimulation. However, for the other 2 subjects, there is little resemblance between the patterns for the two electrode configurations.

A second hypothesis tested in these studies was that low thresholds would be associated with good speech recognition. To test this hypothesis, we examined correlations between mean $\mathrm{T}$ levels across all tested sites in a subject's electrode array and speech recognition in that subject. Across subjects, these mean thresholds varied over a range of $15.3 \mathrm{~dB}$ for $\mathrm{BP}$ stimulation and $12.7 \mathrm{~dB}$ for MP stimulation. Furthermore, there was a moderately strong correlation between mean $\mathrm{T}$ levels for $\mathrm{BP}$ stimulation and those for MP stimulation (fig. 4), suggesting a common underlying cause of this variation across subjects. However, contrary to the hypothesis, we found

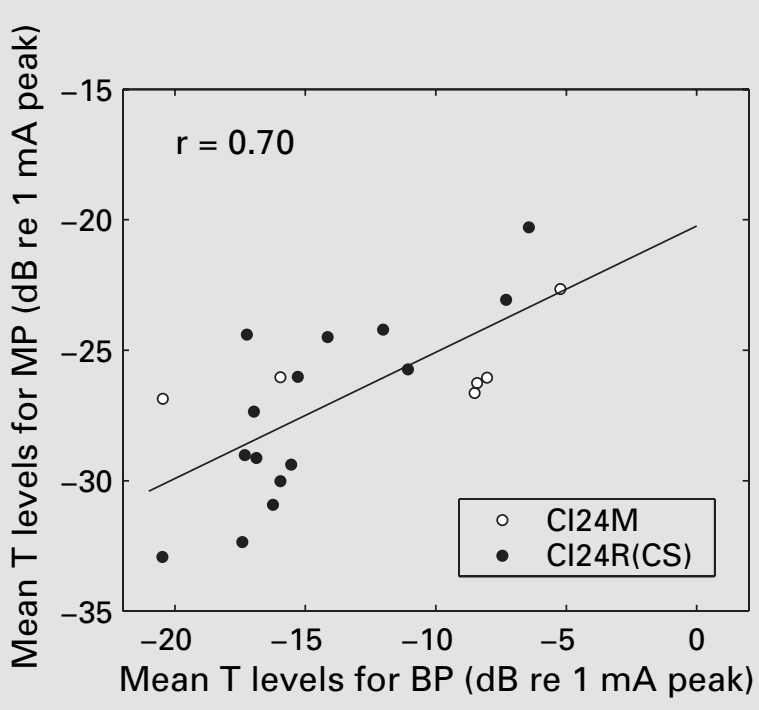

Fig. 4. Relationship between mean $\mathrm{T}$ levels for BP stimulation and mean T levels for MP stimulation. Each data point indicates an individual subject with CI24M implants represented by open circles and CI24R(CS) implants represented by filled circles. A leastsquares regression line for all 21 data points is shown. The correlation coefficient, shown in the upper left corner, is statistically significant ( $\mathrm{z}$ test; $\mathrm{p}<0.05$ ).

Table 2. Correlation coefficients across 21 subjects for each of the six combinations of electrode configuration and type of speech test, using each of the three measures of across-site variation

\begin{tabular}{llll}
\hline & Variance & Range & $\begin{array}{l}\text { Site-to-site } \\
\text { variation }\end{array}$ \\
\hline BP consonants & -0.56 & -0.53 & $(-0.42)$ \\
BP vowels & -0.47 & $(-0.41)$ & $(-0.38)$ \\
BP sentences & -0.52 & -0.44 & $(-0.29)$ \\
MP consonants & -0.70 & -0.66 & -0.59 \\
MP vowels & -0.56 & -0.61 & -0.58 \\
MP sentences & -0.60 & -0.62 & -0.57
\end{tabular}

All correlations were statistically significant ( $\mathrm{z}$ test; $\mathrm{p}<0.05$ ) except for the four marked with parentheses.

no statistically significant correlation ( $\mathrm{z}$ test; $\mathrm{p}<0.05$ ) across subjects between mean $T$ levels and speech recognition for either BP or MP stimulation (fig. 5). Speech recognition performance varied across subjects over a similar range for subjects with high thresholds as it did for subjects with low thresholds. 
Fig. 5. Relationship between mean T levels and speech recognition. Each data point indicates an individual subject with CI24M subjects represented by open circles and CI24R(CS) subjects represented by filled circles. For each subject, the positions on the abscissa represent mean $\mathrm{T}$ levels across all tested sites and positions on the ordinate represent speech recognition scores for that subject. The left and right columns show data for BP and MP stimulation, respectively. The three rows, top to bottom, show data for consonant, vowel, and HINT sentence $(10 \mathrm{~dB} \mathrm{~S} / \mathrm{N})$ tests, respectively. The correlation coefficients are indicated at the lower right corner of each panel. None of these correlations were statistically significant ( $\mathrm{z}$ test; $\mathrm{p}>0.05)$.

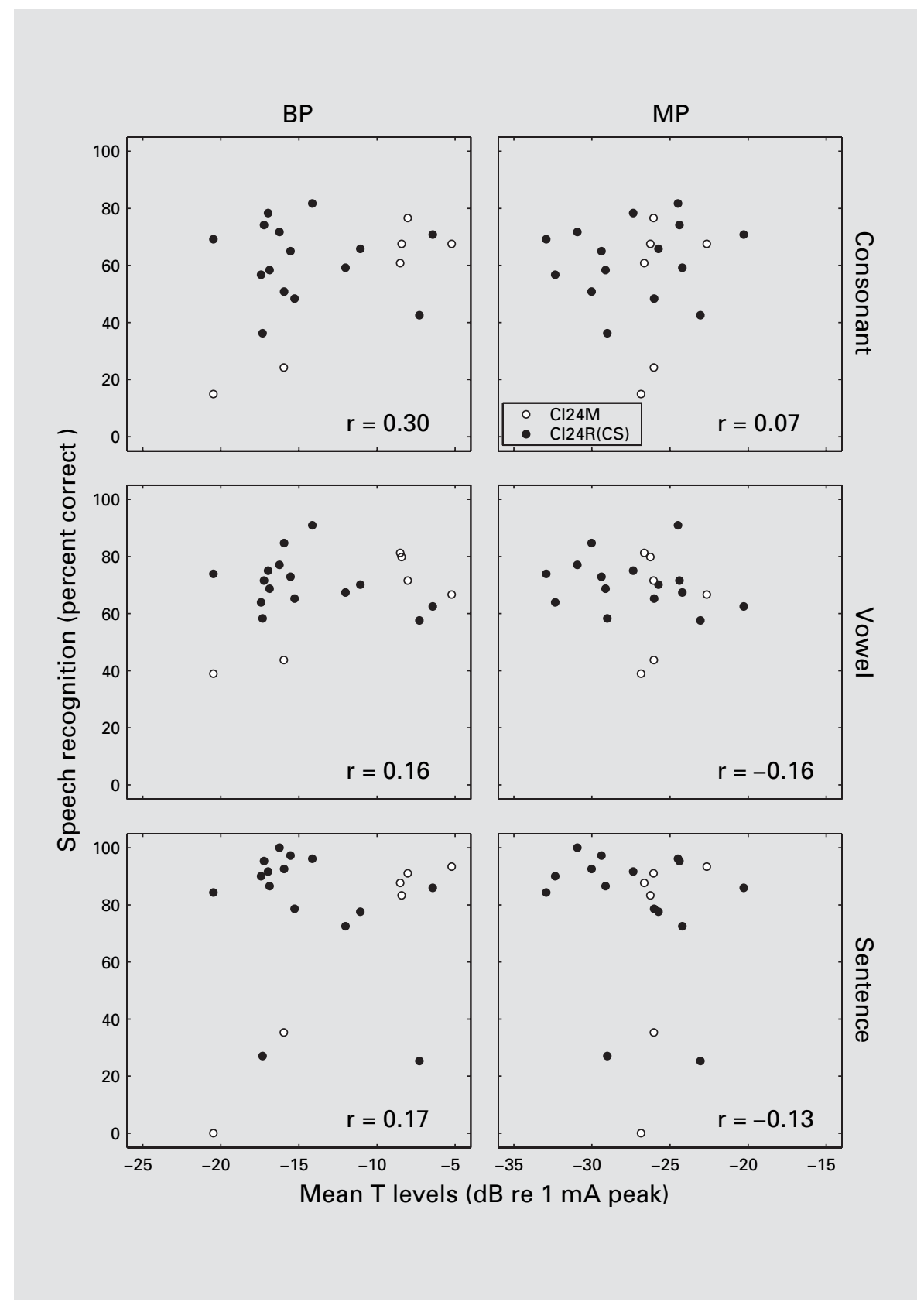

A third hypothesis tested in these studies was that across-site variation in $\mathrm{T}$ levels would depend on the phase duration of the stimulus. We expected to find that the slopes of psychophysical strength-duration functions ( $T$ level versus phase duration functions) would vary across stimulation sites that differed in $\mathrm{T}$ levels for any given phase duration. This expectation was met in a few cases, such as S10 for BP stimulation (fig. 6). In this example, the strength-duration functions for BP stimula- tion for stimulation sites with relatively high $\mathrm{T}$ levels are steeper than those for sites with lower T levels. Thus, the functions tend to converge at longer phase durations and across-site variation is smaller at longer phase durations. For other subjects, such as S20 (fig. 6), the strength-duration functions have nearly the same slopes at all tested sites, so across-site variation in T levels shows little change as a function of phase duration. Figure 7 shows two measures of variation across the 7 or 8 tested sites as a func- 
Fig. 6. Examples of psychophysical strength-duration functions (T-level-versus-phase-duration functions). a, b Functions obtained from 2 CI24M subjects. c, d Functions from 2 CI24R(CS) subjects. The subject number is indicated in the lower left corner of each panel. The solid lines and filled symbols represent data for the MP configuration and the dotted lines and open symbols represent data from the BP configuration. Each line represents data obtained from one electrode site. Typically, strength-duration functions were obtained from 7 or 8 sites in the electrode array.
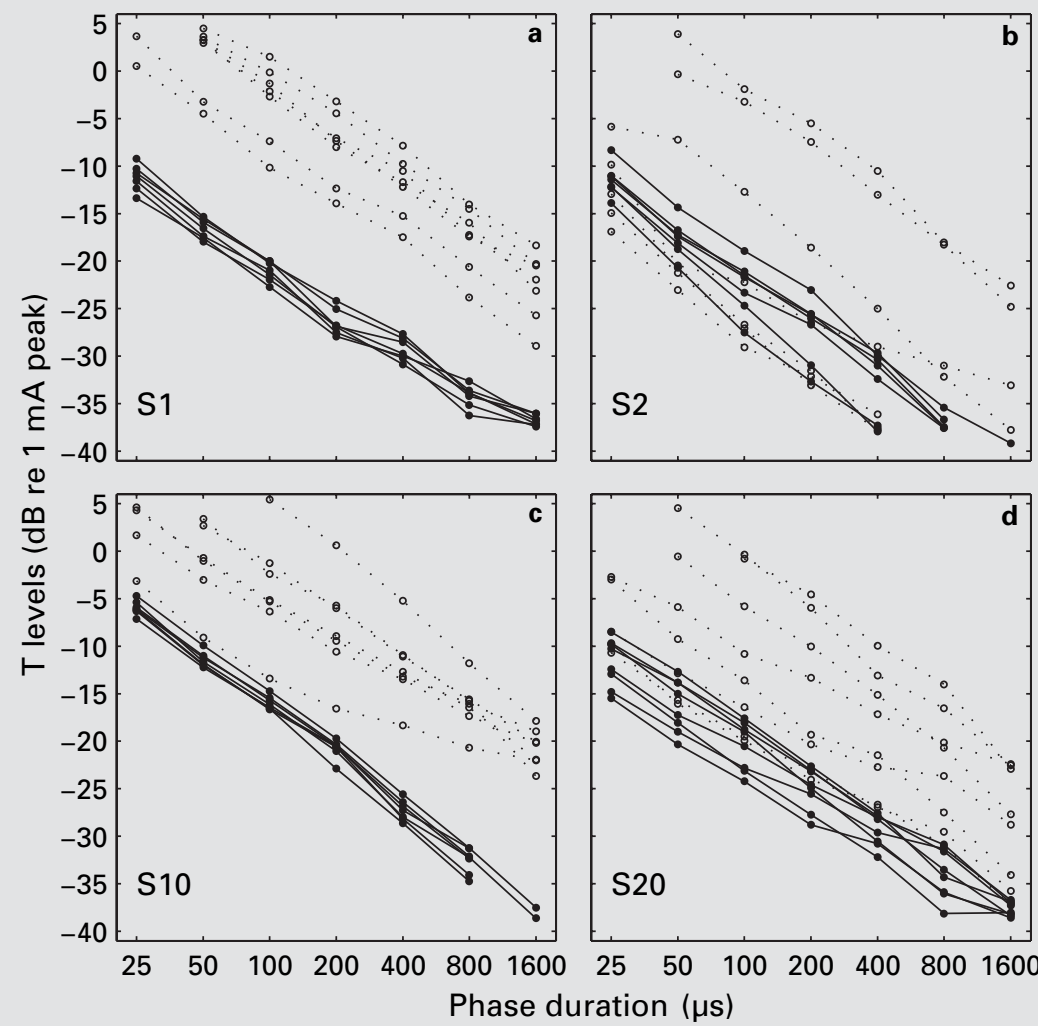

tion of phase duration for all 21 subjects. We found that in the majority of cases, across-site variation showed little change as a function of phase duration (slopes of the functions are near zero). However, in some subjects, acrosssite variation decreased or increased significantly as a function of phase duration in some conditions. Table 3 shows cases in which slopes of the across-site variation versus strength-duration functions shown in figure 7 were significantly different from zero ( $t$ test; $p<0.05$ ). For BP stimulation, 8 of the subjects had statistically significant negative slopes for the across-site range versus phase duration functions and 7 of these 8 subjects also had significantly negative slopes for the across-site variance versus phase duration functions. Thus, for approximately $35 \%$ of the subjects, across-site variation in T levels for BP stimulation decreased as a function of phase duration. Interestingly, all of these subjects had CI24R(CS) implants.

\section{Discussion}

The primary finding in this study was that across-site variation in detection thresholds for both MP and BP stimulation was significantly correlated with speech recognition performance. Subjects with high variation in psychophysical detection thresholds from site to site along the Nucleus 22-electrode scala tympani implant generally had poorer speech recognition than subjects with low across-site variation. This result is consistent with the idea that across-site variation reflects underlying conditions, such as aberrant current pathways and poor nerve survival, that are not compatible with good speech recognition using multichannel stimulation strategies.

The magnitude of across-site variation for MP stimulation was considerably smaller than that for $\mathrm{BP}$, as is commonly observed in the clinic. There may be several mechanisms underlying this effect. One of the most prominent is probably that the voltage drop as a function of distance from the electrodes is much steeper for $\mathrm{BP}$ stimulation than for MP. Thus, with BP stimulation, 


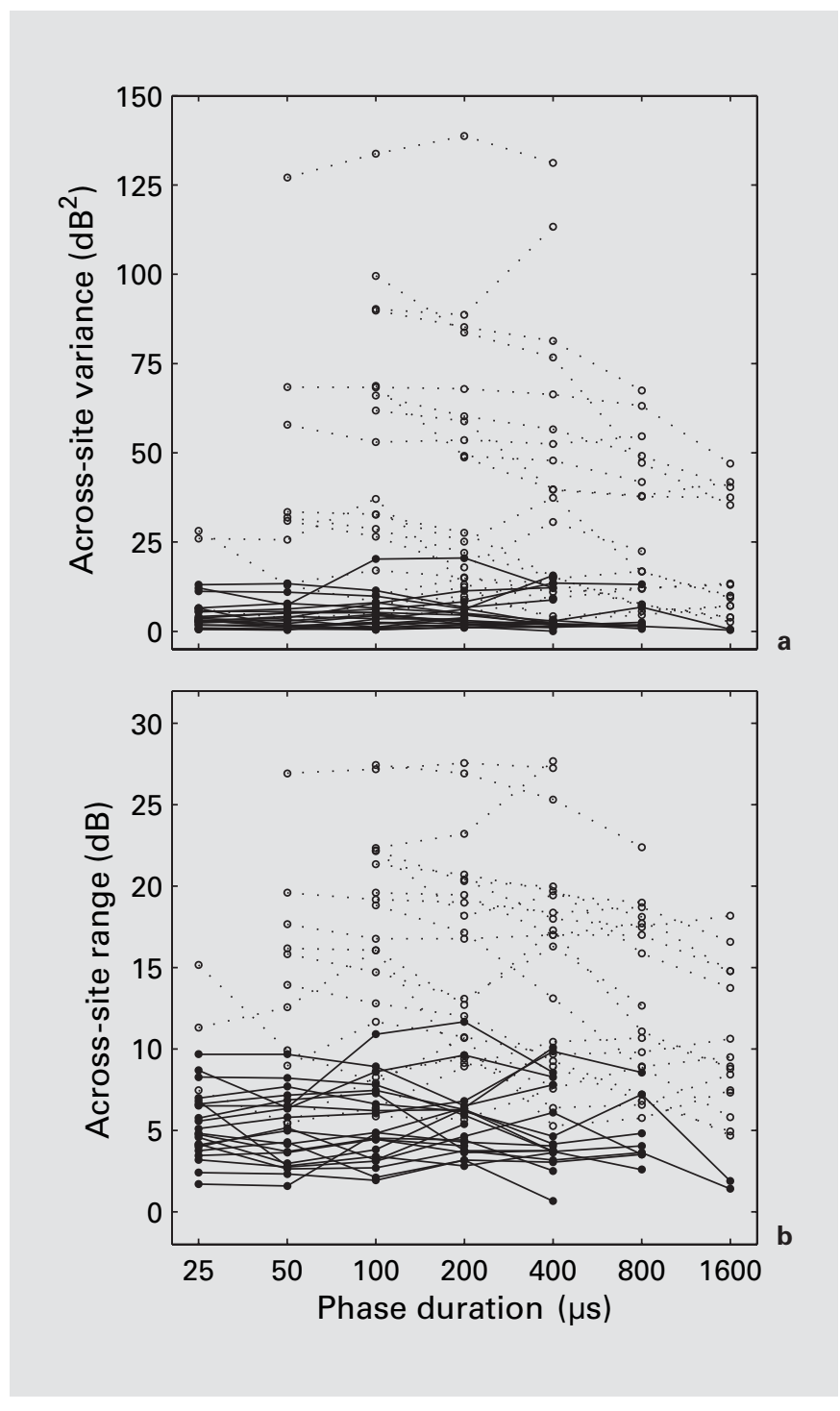

Fig. 7. Across-site variation as a function of phase duration for all the 21 subjects. a On the ordinate, across-site variation is quantified using the variance of the T levels across the 7 or 8 sites. b On the ordinate, the across-site variation is quantified using the range of the T levels across the 7 or 8 sites. In both panels, each line represents data from 1 subject. The solid lines and filled symbols represent data for the MP configuration and the dotted lines and open symbols represent data for the BP configuration. Only data from the phase durations that gave rise to measurable $\mathrm{T}$ levels in all the selected sites were used.

small differences in the distances between the electrodes and the sites of action potential initiation will result in relatively large differences in the amount of current required for activation as compared to the case of MP stimulation.
Table 3. Results of statistical analysis of the slopes of the across-site variation versus phase duration functions shown in figure 7

\begin{tabular}{|c|c|c|c|c|}
\hline Subject & $\begin{array}{l}\text { Across-site } \\
\text { variance for } \\
\text { BP versus } \\
\text { phase } \\
\text { duration }\end{array}$ & $\begin{array}{l}\text { Across-site } \\
\text { variance for } \\
\text { MP versus } \\
\text { phase } \\
\text { duration }\end{array}$ & $\begin{array}{l}\text { Across-site } \\
\text { range for } \\
\text { BP versus } \\
\text { phase } \\
\text { duration }\end{array}$ & $\begin{array}{l}\text { Across-site } \\
\text { range for } \\
\text { MP versus } \\
\text { phase } \\
\text { duration }\end{array}$ \\
\hline \multicolumn{5}{|l|}{$\mathrm{S} 1$} \\
\hline $\mathrm{S} 2$ & & + & & \\
\hline \multicolumn{5}{|l|}{ S3 } \\
\hline $\begin{array}{l}\text { S4 } \\
\text { S5 }\end{array}$ & & + & & + \\
\hline S6 & & - & & - \\
\hline \multicolumn{5}{|l|}{ S7 } \\
\hline \multicolumn{5}{|l|}{ S8 } \\
\hline $\mathrm{S} 10$ & - & + & - & \\
\hline S11 & - & & - & \\
\hline S12 & & - & - & - \\
\hline S13 & - & & - & \\
\hline S14 & - & & - & \\
\hline $\mathrm{S} 15$ & & + & & + \\
\hline \multicolumn{5}{|l|}{ S16 } \\
\hline S17 & - & & - & \\
\hline S18 & & & + & \\
\hline S19 & - & & - & \\
\hline $\mathrm{S} 20$ & - & & - & \\
\hline $\mathrm{S} 21$ & & & & \\
\hline
\end{tabular}

Slopes that were significantly different from zero are marked by + or - signs indicating the direction of the slope.

We found that the correlations between speech recognition and across-site threshold variation for MP stimulation were similar to those for BP stimulation (fig. 2; table 2). However, we found only a weak correspondence within subjects between the across-site patterns of thresholds for the two configurations (e.g. fig. 1) and only a moderate correlation between across-site variance for BP stimulation and that for MP stimulation (fig. 3). This is not surprising because the current paths generated by BP stimulation with respect to the sites of action potential initiation are probably not identical to those generated from the same sites by MP stimulation. Thus, the populations of neurons excited by MP and BP stimulation are probably not identical and this would lead to differences in the amount and pattern of site-to-site variation observed with these two electrode configurations. We interpret the moderate correlation between across-site threshold variance for BP and MP stimulation (fig. 3) and the fact that measures of across-site threshold variation with 
both configurations were correlated with speech recognition (fig. 2; table 2) to indicate that both measures in general reflect the physical and physiological condition of the implanted cochlea. However, currents from these two configurations are probably sampling this variation at different locations in the implanted cochlea and nerve array resulting in less than perfect correspondence between the thresholds obtained with these two configurations.

In contrast to across-site variation in thresholds, mean absolute threshold levels were not correlated with speech recognition (fig. 5). In theory, uniformly low thresholds would indicate good nerve survival and close proximity of the electrodes to the neurons, and these conditions should be favorable for implant performance, including speech recognition. However, uniformly low thresholds are rarely achieved with BP stimulation, even with cochlear implants that are designed to position electrodes close to the modiolus [Pfingst and Xu, 2004; Saunders et al., 2002], and this condition was not seen in any of the subjects in this study. Some of the subjects with good speech recognition had high mean BP thresholds, but low across-site variance. This suggests that consistency from site to site is more important than lower average thresholds.

In this study, the correlations between across-site variation and speech recognition were slightly but consistently stronger for MP stimulation than they were for BP stimulation (table 2). This might be because the speech testing was done with the subject's normal every-day electrode configuration, which was MP. Currently, most cochlear implants are programmed using an MP electrode configuration. The psychophysical detection thresholds that are collected by the audiologist during this process might be useful for estimating the ability of the patient to acquire good speech recognition and for selecting the postimplantation course of therapy.

In many subjects, we found little effect of stimulus phase duration on across-site variance (fig. 7; table 3 ). The most common exception to this result occurred in about one third of the cases with BP stimulation where acrosssite variation decreased significantly as a function of phase duration. For MP stimulation, we saw little effect of phase duration on across-site variation. These findings are convenient with respect to clinical assessment because shorter-duration pulses and monopolar stimulation are typically used in most contemporary processing strategies and thresholds for these parameters would be commonly obtained in the clinic.

Multiple mechanisms may underlie the across-site variability in psychophysical detection thresholds ob- served in this study. Because detection of electrical stimuli is a simple process that does not require complex cognitive skills, it is reasonable to assume that variation in detection thresholds across stimulation sites results from relatively simple physical and/or physiological variables in the implanted cochlea and in the auditory neurons. Much of the across-site variability in T levels could be explained by variability in the distances from the electrodes to the sites of action potential initiation. This variability in turn could be due to several factors, including variation in the location of electrodes in the scala tympani with respect to the modiolar wall, variation in current paths from the electrodes to the neurons due to fibrous tissue and new bone formation in the implanted scala, and neuropathology. These variables could affect speech recognition with cochlear implants for several reasons.

Aberrant current paths created by tissue growth in the scala tympani or by pathology of neurons near the electrodes could result in significant distortion of the intended frequency to place map, which is a key element of the auditory prosthesis stimulation strategy. Distortions in the frequency to place map created by neuropathology have been modeled in studies using acoustic simulations of auditory prosthesis processors and by experiments in subjects with cochlear implants [Shannon et al., 2002]. In these studies, 'holes in hearing' were created by either omitting the information from selected analysis filters or by reassigning the information from the 'hole' region to areas surrounding the holes. It was found that small holes had only small effects on sentence recognition. Hole sizes of 4 or more adjacent electrodes $(>3 \mathrm{~mm})$ were required to produce reductions in sentence recognition of $>20 \%$. Only one hole was tested at a time, so it may be that more severe effects would be seen with multiple holes. In addition, we must consider that other variables may be contributing to the deficit seen in cochlear implant users with very poor speech recognition. One possible factor, related to across-site threshold variation, would be a cross-site variation in the perception elicited by each stimulation site. It is believed that electrodes that are positioned closer to the sites of action potential initiation produce narrower spatial patterns of neural excitation at a given loudness level than do electrodes that are remote from the excitable neurons. If one site elicits a broad excitation pattern, and an adjacent site elicits a narrower excitation pattern, this perception may be confusing to the listener.

The across-site threshold variation data for individual subjects might be useful clinically in identifying the sources of poor speech recognition for some subjects and in pointing to possible remedies. If the site-to-site variation 
is itself disruptive, it might be possible to reduce this variation by omitting certain channels from an individual's processor program. This idea remains to be tested in this specific context. However, it has previously been demonstrated that omitting channels with poor electrode discrimination can lead to improved speech recognition [Zwolan et al., 1997].

Molecular biological techniques are currently being developed for regulation and control of variables such as tissue growth in the implanted cochlea and spiral ganglion cell death and regrowth [Miller et al., 2002]. Thus, in future years, it might be possible to control some of the biological variables that lead to large across-site variation in detection thresholds and thus, perhaps, control the decrements in speech recognition performance that are correlated with high across-site threshold variability.

\section{Acknowledgements}

This work was supported by NIH/NIDCD grants R01 DC 03808 and R01 DC 03389. Our thanks go to the human subjects who participated in this research.

\section{References}

Blamey PJ, Arndt P, Bergeron F: Factors affecting auditory performance of postlingually deaf adults using cochlear implants. Audiol Neurootol 1996;1:293-306.

Colombo J, Parkins CW: A model of electrical excitation of the mammalian auditory-nerve neuron. Hear Res 1987;31:287-311.

Hillenbrand J, Getty LA, Clark MJ, Wheeler K: Acoustic characteristics of American English vowels. J Acoust Soc Am 1995;97:30993111.

van den Honert C, Stypulkowski PH: Physiological properties of the electrically stimulated auditory nerve. II. Single fiber recordings. Hear Res 1984; 14:225-243.

Kawano A, Seldon HL, Clark GM, Ramsden RT, Raine $\mathrm{CH}$ : Intracochlear factors contributing to psychophysical percepts following cochlear implantation. Acta Otolaryngol (Stockh) 1998; 118:313-326.

Kileny PR, Zimmerman-Phillips S, Kemink JL, Schmaltz SP: Effects of preoperative electrical stimulability and historical factors on performance with multichannel cochlear implant. Ann Otol Rhinol Laryngol 1991;100:563568
Knutson JF, Hinrichs JV, Tyler RS, Gantz BJ, Schartz HA, Woodworth G: Psychological predictors of audiological outcomes of multichannel cochlear implants: Preliminary findings. Ann Otol Rhinol Laryngol 1991;100:817822.

Miller JM, Miller AL, Yamagata T, Bredberg G, Altschuler RA: Protection and regrowth of the auditory nerve after deafness: Neurotrophins, antioxidants and depolarization are effective in vivo. Audiol Neurootol 2002;7:175-179.

Nilsson M, Soli SD, Sullivan JA: Development of the Hearing in Noise Test for the measurement of speech reception thresholds in quiet and in noise. J Acoust Soc Am 1994;95:1085-1099.

Pfingst BE: Auditory prostheses; in Chapin JK, Moxon KA (eds): Neural Prostheses for Restoration of Sensory and Motor Function. Methods and New Frontiers in Neuroscience. Boca Raton, CRC Press, Inc, 2000, pp 3-43.

Pfingst BE, Xu L: Across-site variation in detection thresholds and maximum comfortable loudness levels for cochlear implants. J Assoc Res Otolaryngol 2004;5:11-24.
Rubinstein JT, Parkinson WS, Tyler RS, Gantz BJ: Residual speech recognition and cochlear implant performance: Effects of implantation criteria. Am J Otol 1999;20:445-452.

Saunders E, Cohen L, Aschendorff A, Shapiro W, Knight M, Stecker M, Richter B, Waltzman S, Tykocinski M, Roland T, Laszig R, Cowan R: Threshold, comfortable level and impedance changes as a function of electrode-modiolar distance. Ear Hear 2002; 23:28S-40S.

Shannon RV, Galvin JJ 3rd, Baskent D: Holes in hearing. J Assoc Res Otolaryngol 2002;3:185199.

Shannon RV, Jensvold A, Padilla M, Robert ME, Wang X: Consonant recordings for speech testing (letter). J Acoust Soc Am 1999;106:7174.

Skinner MW, Arndt PL, Staller SJ: Nucleus 24 advanced encoder conversion study: Performance vs preference. Ear Hear 2002;23:2S$25 \mathrm{~S}$

Zwolan TA, Collins LM, Wakefield GH: Electrode discrimination and speech recognition in postlingually deafened adult cochlear implant subjects. J Acoust Soc Am 1997;102:3673-3685. 